\title{
Personalized Remote Monitoring of the Atrial Fibrillation Patients with Electronic Implant Devices
}

\author{
Gokce B. Laleci ${ }^{1 *}$, Asuman Dogac ${ }^{1,2}$, Mustafa Yuksel ${ }^{1,2}$, Yildiray Kabak ${ }^{1}$, Elena \\ Arbelo $^{3}$, Franz Danmayr ${ }^{4}$, Lynne Hinterbuchner ${ }^{4}$, Catherine Chronaki ${ }^{5}$, Marco \\ Eichelberg $^{6}$, Christian Lüpkes ${ }^{6}$ \\ ${ }^{1}$ Software Research and Development and Consultancy Ltd., Ankara, Turkey \\ ${ }^{2}$ Dept. of Computer Eng., Middle East Technical University, Ankara, Turkey \\ ${ }^{3}$ Hospital Clinic I Provincial de Barcelona, Barcelona, Spain \\ ${ }^{4}$ Salzburger Landeskliniken BetriebsgesmbH, Salzburg, Austria \\ ${ }^{5}$ Foundation for Research and Technology Hellas - Institute of Computer Science, \\ Institute of Computer Science, Crete, Greece \\ ${ }^{6}$ OFFIS e. V., Institute for Information Technology, Oldenburg, Germany
}

Submitted July 2010. Accepted for publication December 2010.

\begin{abstract}
Cardiovascular Implantable Electronic Devices (CIED) are gaining popularity in treating patients with heart disease. Remote monitoring through care management systems enables continuous surveillance of such patients by checking device functions and clinical events. These care management systems include decision support capabilities based on clinical guidelines. Data input to such systems are from different information sources including medical devices and Electronic Health Records (EHRs). Although evidence-based clinical guidelines provides numerous benefits such as standardized care, reduced costs, efficient and effective care management, they are currently underutilized in clinical practice due to interoperability problems among different healthcare data sources. In this paper, we introduce the iCARDEA care management system for atrial fibrillation patients with implant devices and describe how the iCARDEA care plan engine executes the clinical guidelines by seamlessly accessing the EHR systems and the CIED data through standard interfaces.
\end{abstract}

Keywords: electronic health records, care management, remote monitoring of cardiac patients

*Corresponding Author: Gokce B. Laleci, SRDC Ltd., Ankara, Turkey. Phone: (90) 312 2101763. Fax: (90) 312 2101837. E-mail: gokce@srdc.com.tr. Other authors: asuman@srdc.com.tr; mustafa@srdc.com.tr; yildiray@srdc.com.tr; elenaarbelo@secardiologia.es; F.Danmayr@salk.at; 1.hinterbuchner@salk.at; chronaki@ics.forth.gr; eichelberg@offis.de; Christian.Luepkes@Offis.de. 


\section{INTRODUCTION}

Management of cardiac arrhythmia abnormalities that are not transient or reversible requires constant clinical monitoring as a chronic condition. Delays on diagnosis or medical assistance increase the risk of adverse outcomes such as heart failure, stroke, and sudden cardiac death. Therefore, Cardiovascular Implantable Electronic Devices (CIED) have become a part of the standard therapy in patients who are at risk of lifethreatening cardiac arrhythmias.

According to a consensus statement prepared jointly by the Heart Rhythm Society and the European Heart Rhythm Association [1], more than 800,000 patients in Europe have implanted CIEDs for the treatment or secondary prevention of cardiac arrhythmias. In addition, the number of follow-up visits for patients with implanted cardiac devices (ICDs) exceeds 5.8 million per year, and that number will continue to increase as more devices are implanted. The exponential growth rate of cardiac device implantation calls for new methods of long-term surveillance with a view to optimizing patient safety and care, alleviating the burden of caregivers, and lowering health care costs through information communication technology support [2].

CIED devices with remote monitoring capabilities can store and transmit the cardiac status and device function data. Remote sensor devices are located in patients' homes to transfer stored data from the cardiac implant to a remote monitoring service center. These remote monitoring service centers operated by device manufacturers, receive, store, analyze and translate transmitted data into patient-specific reports and allow healthcare professionals to access patient data or to receive alerts in case of unusual persisting data variations. However, some limitations of these remote monitoring service centers exist:

- Access to follow-up information transmitted via CIEDs often requires clinicians to use multiple vendor specific systems and interfaces, as well as manual and paper-based processes, thus complicating or compromising efficiency and quality of clinical workflows.

- CIED data are neither integrated with the healthcare processes, nor with Electronic Healthcare Record (EHR) systems of the healthcare provider. As a result, the analysis and the alerts only address the CIED data, and hence complex decision making requiring the past medical history and current health status of the patient cannot be performed by the remote monitoring service centers.

The iCARDEA project [3] was set out to establish an interoperability framework to semi-automate the follow-up of cardiac arrhythmia patients with care plans based on computer interpretable clinical guideline models by seamlessly accessing the CIED data and the EHRs of the patients. In the iCARDEA architecture, the "IHE Implantable Device Cardiac Observation Profile (IDCO)" [4] is employed to automatically expose CIED data from different vendors in a machine processable format to be used in the care plan of the patients. While executing care plans for monitoring CIED patients with Atrial Fibrillation (AF), further information from the patient EHRs is needed, such as the history of the non-cardiac conditions, detailed information about the severity of each condition (e.g., record of prior hospitalizations, and details of therapy for the 
condition), the medications taken at the time of spontaneous arrhythmia occurrence, and the non-cardiac conditions denoting contraindications to the proposed therapies in order to decide the optimum medical therapy for a specific patient. The major challenge addressed in accessing the EHR systems is the interoperability problem of communicating with various heterogeneous EHR systems. To be able to avoid routinely monitoring a wide variety of clinical data from disparate systems, and developing ad hoc interfaces to access heterogeneous systems, IHE has specified the "Care Management Profile" [5] which is employed in the iCARDEA system.

In iCARDEA, the care plans are designed based on clinical guidelines. Clinical guidelines include plans for treatment and aim to reduce inter-practice variations and the cost of the medical services, to improve the quality of care, and to standardize clinical procedures [6]. Although there are guidelines for management of AF [7] and for monitoring of cardiovascular implantable electronic devices [8], respectively, guidelines for semi-automatic management of CIED patients with AF problem do not exist. For this reason, one of our first activities in iCARDEA Project was analyzing the available clinical guidelines for the management of AF and also monitoring of CIED data, as well as designing flowcharts to semi-automate the remote monitoring of AF patients through CIED devices while accessing their EHRs. Several computer interpretable models of clinical guidelines [9] have been proposed so far. In the iCARDEA system, GLIF [10] is employed for the definition of the care plans and an engine is developed to execute them.

The aims of this paper are (a) to briefly introduce the interoperability architecture offered by the iCARDEA system for remote monitoring of CIED Patients in integration with EHR systems, and (b) to present the management of AF care plan demonstrating how iCARDEA interoperability architecture enables semi-automatic execution of this care plan as a guidance to clinicians through full and seamless access to the CIED and EHR data. A case study will be realized in Austria in clinical settings after the implementation of iCARDEA architecture is finalized.

This paper is organized as follows: In Section 1.1, the related work in this domain is presented emphasizing the required improvements. In Section 2, the overall system architecture is introduced, while in Section 3, the execution of a sample care plan for the management of $\mathrm{AF}$ is presented by demonstrating how it accesses the data sources seamlessly based on standard interfaces. In Section 4, discussion and comparison with published work are presented. Finally Section 5 concludes the paper and presents future work.

\subsection{Related Works}

There are a number of pilot studies in the literature that address remote monitoring of cardiac patients with electronic implant devices through the facilities provided by CIED manufacturers. A good survey of several studies for remote monitoring of cardiovascular implantable electronic devices was presented in [11] by discussing the advantages, the privacy and legal issues. Clinical experiences of remote monitoring for "early detection of device technical troubles and differential diagnosis of appropriate and inappropriate discharges" were presented in [12]. It was stated that the morbidity 
of patients with ICD is closely related to the appropriateness of ICD therapies as well as technical problems of the device, and early detection and handling of these adverse events might significantly decrease morbidity in ICD patients. Several studies concluded that remote monitoring allows early detection of medical and technical events in ICD patients, improves individualized therapy control, and shortens physicians' reaction time to arrhythmias and technical problems [e.g., 15-17].

A clinical case study on home monitoring of an ICD patient with incessant ventricular tachycardia demonstrated the need to access patient's demographics, current problems, allergies and current medication in addition to the electrophysiological data that can be collected from CIEDs [18]. Accessing these patient's clinical data is required to adjust the medication of the patient through detection of incessant ventricular tachycardia for cardiomyopathy problem. In this case, the remote monitoring capability of a CIED enabled the physician to view the alerts generated by the CIED through the CIED portal, and the rest was a manual process for the physician to examine the report and to call the patient for an in-clinic follow-up. The doctor then adjusted the doses of the patient's current medication based on EHR, and an antiarrhythmic drug treatment was decided.

A number of tele-monitoring systems were presented for predicting mortality and rehospitalization in heart failure patients [19]. Although this study does not address the requirement of accessing EHRs for its decision mechanism, the European Society of Cardiology guidelines for the diagnosis and treatment of acute and chronic heart failure [20] lists several laboratory results (e.g., elevated uric acid) and clinical conditions (e.g., sleep-related breathing disorders) as possible predictors for the prognosis of heart failure. Through a system like iCARDEA, it would be possible to incorporate these EHR data into the decision making process in the tele-monitoring system.

\section{ICARDEA SYSTEM ARCHITECTURE}

The iCARDEA system aims to automate the follow-up of cardiac arrhythmia patients with implanted CIED devices through computer interpretable clinical guideline models using standard device interfaces and integrating patient EHRs. Figure 1 shows the overall architecture and the environment in which iCARDEA needs to provide interoperation services. The major components of the system are as follows:

1. Adaptive Care Planner for the CIED Recipients: In the iCARDEA project, the personalized follow-up of CIED patients is coordinated through a "care plan" which is an executable definition of computer interpretable clinical guideline models. The care plans are represented in GLIF, and the care plan engine is capable of semi-automatically executing the care plan by processing its machine processable definition. The control flow of the care plan is dynamically adapted based both on the patient's context derived from the data from CIEDs and the medical context from the EHRs. In this way, the execution of the care plan is personalized. Through a graphical monitoring tool, the physicians are allowed to follow the execution of the care plan in detail, and to coordinate the flow of actions when consultations to physicians are required.

2. The CIED Data Exposure Module: This module uses "IHE Implantable Device Cardiac Observation Profile (IDCO)" to expose the CIED data from different 


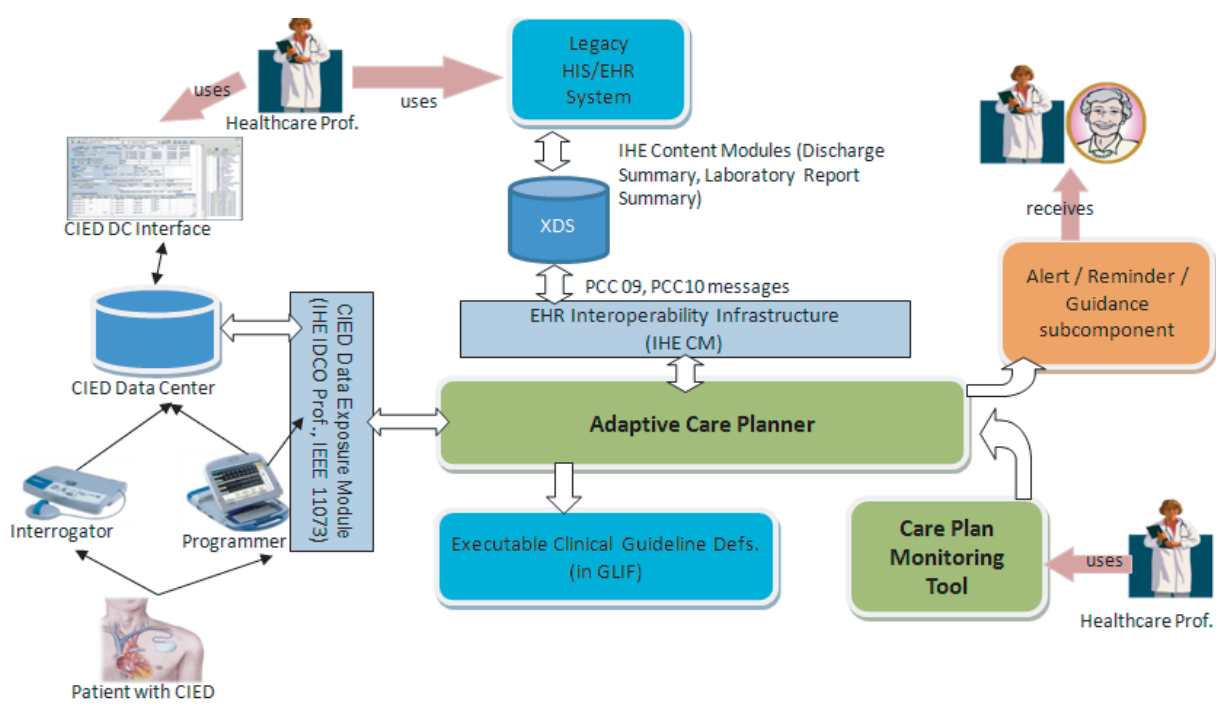

Figure 1. iCARDEA architecture overview

vendors in a machine processable format to be used in the care plans of the patients, through a component that allows accessing the CIED portal of the vendor and triggers the CIED data export automatically from the CIED data center (periodically every $\mathrm{x}$ hours or each morning at a defined time). The CIED Data Listener Component waits for the exported data by either scanning a configurable directory in case data is exported directly to a vendor system in the clinic, or listening to a pre-configured port for the exported data using the IHE IDCO/HL7 v2.5 protocol in case of direct network retrieval. In both cases, the reports received from CIED data centers as PDF file(s) need to be processed to extract the structured CIED data. The Data Translation Service subsystem creates a valid IHE IDCO format (HL7 v2.5 ORU Message) and makes the structured CIED data available to the iCARDEA adaptive care planner through PCD-09 Send Observation transaction.

3. EHR Interoperability Infrastructure: In order to execute the clinical guidelines, it is necessary to have access to medical history of the patients in the EHR systems. Considering that there are various EHR systems with proprietary interfaces, in iCARDEA, the "IHE Care Management (CM) Profile" is used. In our system, the proprietary hospital information systems export "Discharge Summary" and also "Laboratory Report Summary" CDA (HL7 Clinical Document Architecture [21]) documents in conformance to IHE CDA document templates [22] to an EHR server which is implemented as an IHE Cross Enterprise Document Sharing (XDS) Repository [23]. This EHR server also acts as a "Clinical Data Source" by implementing the IHE CM profile. In this way, the Adaptive Care Manager can subscribe to update notifications for the clinical data needed to execute the care plans. The IHE Care Management profile specifies standard interfaces to extract these data from the EHR systems. The two standardized transactions used in the iCARDEA system are as follows: 
- "PCC-09 Care Management Data Query" allows querying the clinical data sources such as the EHR systems for the data required to execute the care plan.

- "PCC-10-V3 Care Management Update" allows the clinical data sources (EHR systems) to send the updated clinical data to the subscribed care management systems as an HL7 V3 message.

Additionally, IHE has specified "Content Modules" to be used as the payloads of these transactions to transfer clinical data [22] in terms of CDA Sections and Entries. The HL7 Clinical Document Architecture (CDA) is a document markup standard that specifies the structure and semantics of "clinical documents" for the purpose of exchange; each CDA document is made up of CDA Sections and each Section is made up of CDA Entries. Different content module templates for CDA documents such as Discharge Summary and Referral Summary; CDA Sections such as History of Present Illness, Medications, and CDA Entries such as Problem Entry, Vital Signs Observation have been specified.

While a care manager queries a clinical data source, it specifies the type of the clinical data required through a code specified in the "careProvisionCode" field, such as "LABCAT", meaning all lab results. For each code specified in this controlled code list, the IHE content module template (for example "Simple Observations" template is specified for reporting lab results) is also specified through which the clinical data update is to be sent. The clinical data sources send the updated clinical data to the iCARDEA care plan engine by conforming to these content module templates. In this way, the interoperability of the transactions among clinical data sources and care managers is guaranteed.

\section{A SAMPLE CARE PLAN EXECUTION}

In this section, the execution of the iCARDEA care plan engine is described through an example care plan developed for the management of AF for CIED patients. The care plan includes three clinical guidelines: the first guideline is for management of patients with $\mathrm{AF}[7]$, the second for the monitoring of cardiovascular implantable electronic devices[8], and the third for the management of patients with ventricular arrhythmia and the prevention of sudden cardiac death[24]. The care plan emphasizes oral anticoagulation therapy as a basic point in the therapy of AF that should be administered after the early recognition of this arrhythmia for preventing thromboembolic events like stroke. The iCARDEA adaptive care plan engine continuously monitors the CIED patients and provides guidance to the physician for a reliable and fast decision on whether to start anticoagulation therapy by checking the alerts sent by the CIED devices, together with the patient's medical history, current medications, recent lab results, and possible contraindications. The clinicians of Salzburg University Hospital, and Hospital Clínic de Barcelona have developed the flowcharts of the care plan by examining the selected clinical guidelines, and these flowcharts are a computer interpretable care plan definition in GLIF.

\subsection{Triggering of the Care Plan Execution through a CIED Event}

This study has received approval by the Research Ethics Committee of Salzburg University Hospital. In this case, after the CIED is implanted, the patient is sent home, and his condition is continuously monitored through the iCARDEA system. As presented in Figure 2, the care plan is initiated whenever an AF event is detected by the 


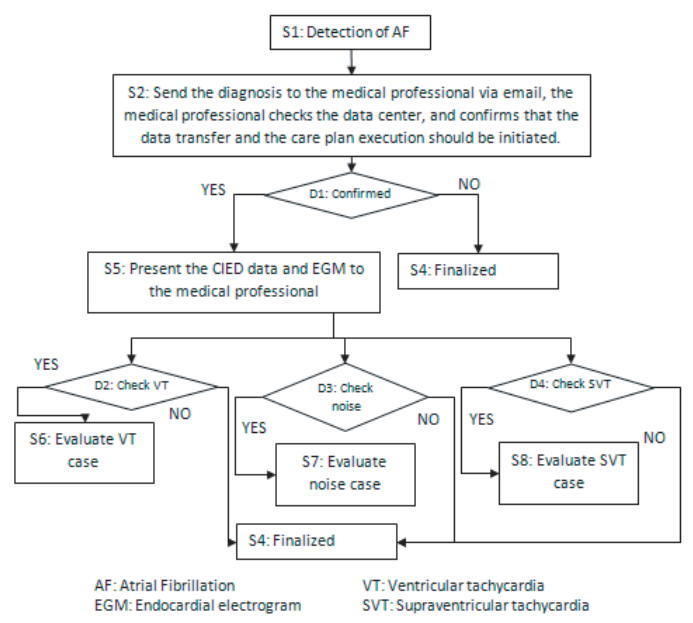

Figure 2. Initiation of care plan by detection of an $\mathrm{AF}$ event

CIED indicating that the AF daily burden has exceeded the specified threshold (S1). Whenever such an event is detected, the physician is notified automatically through an instant message by the CIED system, and the physician can check the current measurements and the Endocardial Electrogram (EGM) (S2, S5) through the CIED data center portal. If necessary, the execution of care plan can be initiated. In the meantime, the recent CIED data together with EGM have already been transferred to the Adaptive Care Planner through the PCD-09 Send Observation transaction in conformance to IHE IDCO Profile. After each abnormal situation detected by CIEDs, or after routine remote follow-ups, the CIED data are sent to the Adaptive Care Planner through the PCD-09 Send Observation transaction in conformance to IHE IDCO Profile, with the help of iCARDEA CIED data Exposure Module. These CIED Data are stored in the database of the Adaptive Care Planner to be accessed while the care plan is executed.

As an initial step, the care plan checks the computer interpretable CIED data received to identify if it is a "Ventricular Tachycardia case (VT)", a "Supraventricular Tachycardia case (SVT)", or just a "Noise" (D2-D4). All of these decisions are presented to the physician through a graphical interface to be confirmed by checking the EGM when necessary. Based on this decision, different treatment options are followed.

For example, in the case of a "Noise" detection (Noise is a non-cardiac signal produced in the device's sensing channels, which may be misinterpreted as cardiac electrical activity), by processing the previously retrieved CIED data, the care plan checks "the number of inappropriate discharges" and is confirmed with the physician (Figure 3, D6). Inappropriate discharges are the shocks delivered for reasons other than life threatening arrhythmia. If there have been several such inappropriate discharges, the care plan recommends "immediate referral to the clinic" (R3); if there has been only one such inappropriate discharge, the care plan recommends "to arrange a follow-up in 


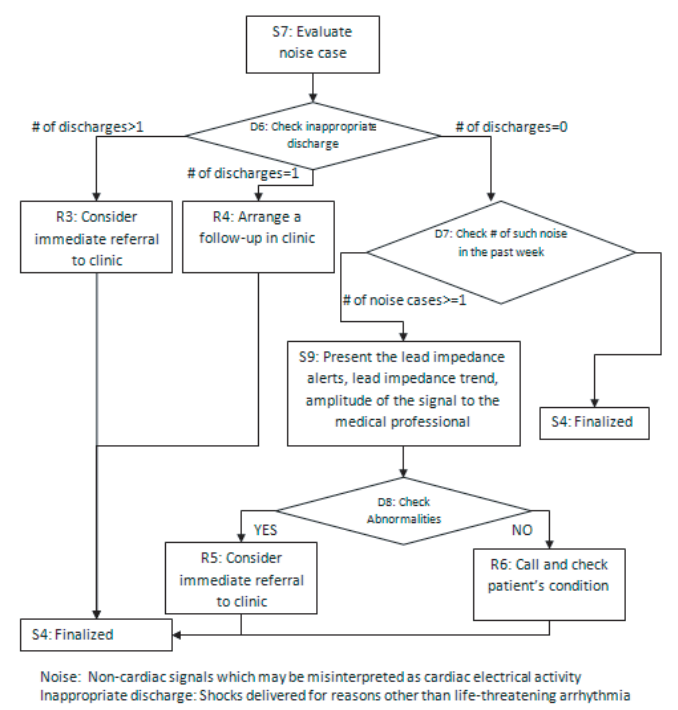

Figure 3. Evaluating the noise case

the clinic" (R4); and if there has been no such inappropriate discharge, the care plan engine checks the occurrence of such noise in the past week for the abnormalities in lead impedance values, lead impedance trends and amplitude of the signal from its database (D7, S9). If such abnormalities are detected, the care plan engine recommends "immediate referral to the clinic" to re-program the CIED device (R5).

In "Supraventricular Tachycardia (SVT) case" (Figure 4, S8), the care plan engine evaluates the case of "Sinus Tachycardia", "Real Supraventricular Tachycardia", and "Real AF", incorporating confirmation from the physician (Figure 4, D9-D11).

\subsection{Accessing the Electronic Healthcare Records to Control the Flow of Action in the Care Plan}

In the "Real AF" case, as shown in Figure 4, if there were no "inappropriate discharges", the possibility of "Anticoagulation Therapy" is considered (D14). For this decision, the care plan engine needs to access the EHRs of the patient. First of all, the CHADS2 score [25] of the patient should be checked to decide whether anticoagulation therapy is needed (D15). The CHADS2 score is a guideline approved risk score for thromboembolic events in patients with $\mathrm{AF}$, which provides guidance to the physician about the necessity for oral anticoagulation therapy. CHADS2 score is an acronym of the risk factors (Congestive heart failure, Hypertension, Age, Diabetes, Stroke in clinical history). Each risk factor is assessed with a value of 1, whereas existence of stroke is valuated as 2 . If the sum of these values is higher than 1 , it indicates the oral anticoagulation therapy. If CHADS2 score is equal to 0 , then the care plan engine recommends "Prescription of 81-325 mg Aspirin daily" (R13a). If the CHADS2 score 


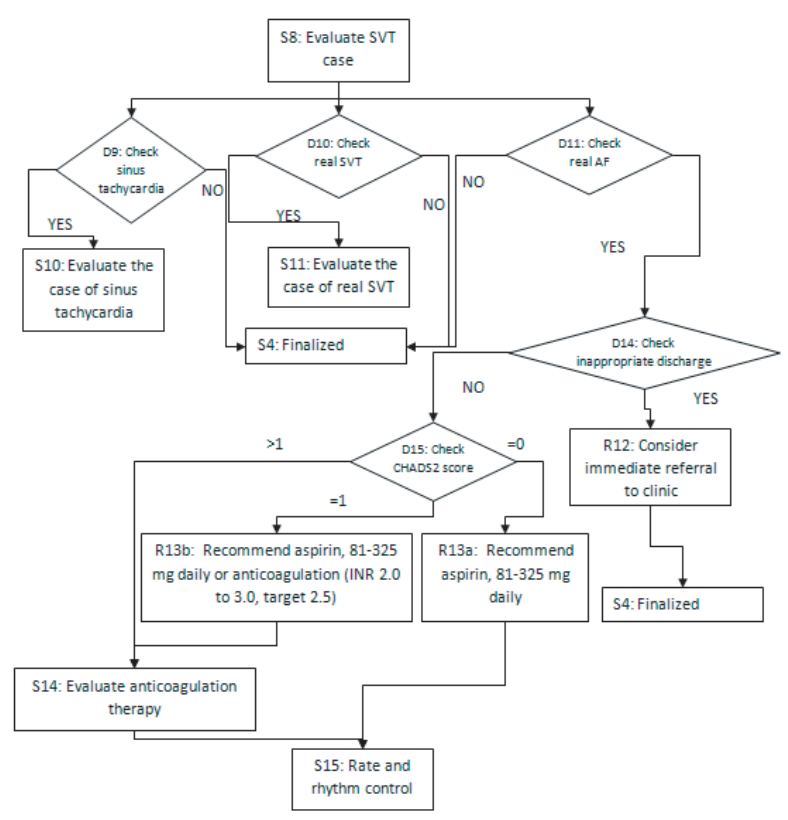

Figure 4. Evaluating the SVT case

is equal to 1, besides Aspirin therapy, Anticoagulation therapy can also be considered as an option (R13b).

When the care plan is personalized for a specific patient and registered in the care plan engine, the machine processable care plan definition represented in GLIF is processed, and the clinical data to be retrieved from the EHR server are identified. In our care plan, each one of such cases is represented through a "GetDataAction" in the GLIF definition where the required clinical data are coded both by the CDA Entry semantics (e.g., specifying the SNOMED CT code for "Congestive Heart Failure" as "42343007") and the CDA Section semantics (e.g., specifying the LOINC code for "History Of Past Illness" as "11348-0"). The Adaptive care plan engine processes these definitions and creates a "PCC-09 Clinical Data Query" to be sent to the EHR Server. For instance, in the present case, the care plan engine first checks the IHE Content Module coded with the LOINC code "11348-0" which corresponds to "History of Past Illness Section" template [26]. This template contains a list of CDA "Problem Concern Entries", all of which must be retrieved from the EHR system. Therefore, the care plan engine creates a "Clinical Data Query" specifying the "careProvisionCode" as "PROBLIST" to retrieve the updates for "All of the Problem Concerns".

As a response to this query, the clinical data source, the EHR server in our case, presents an initial response, providing the registered Problem Concern List for this specific patient in conformance with the specified "Problem Concern" Entry Template [27]. The adaptive care plan engine is kept up to date with the historical and current 


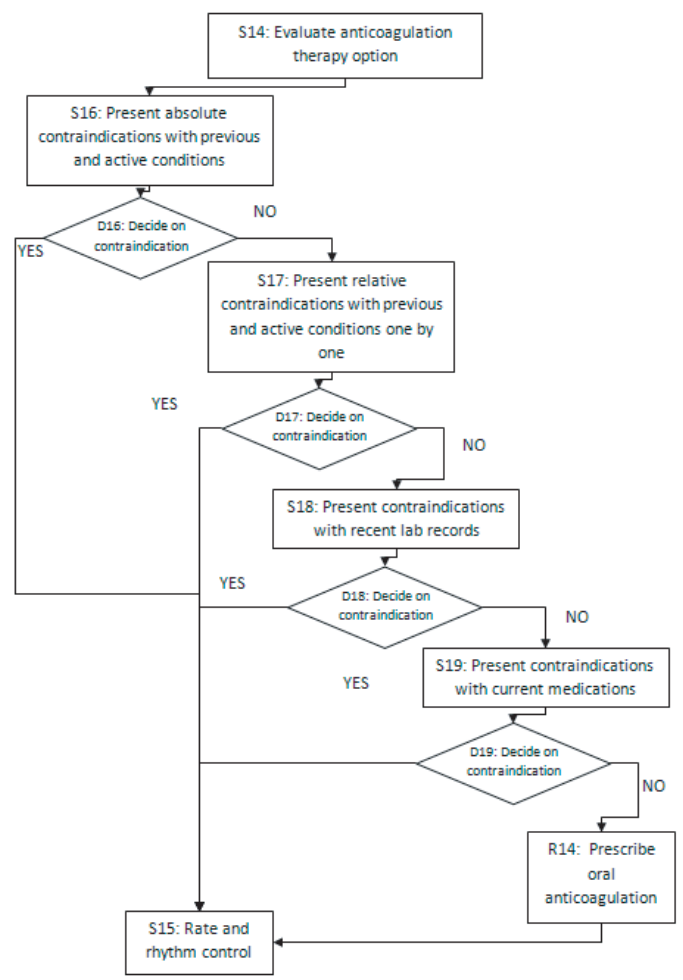

Figure 5. Checking contraindications for anticoagulation therapy

clinical problems of the patient. Whenever such "PCC V3 Care Management Update" messages are received, they are processed and stored in the database of the care plan engine to be retrieved when the care plan is executed.

After retrieving the problem concern entries from the EHR system as mentioned above, the CHADS2 score is calculated and the care plan engine checks if it is appropriate to prescribe anticoagulation drugs for the patient. The contraindications of anticoagulation drugs with the previous and active conditions of the patient, including active medications and recent lab results, need to be checked (Figure 5, S16-S19). For instance, prescribing anticoagulation drugs for patients with "Gastrointestinal bleeding" is not recommended, and likewise if a recent lab report showed the "erythrocyte count" out of specified thresholds, indicating probable "Blood dyscrasia". Finally, prescription of anticoagulation drugs should not be considered as a concomitant drug if the patient is already on medications such as "Hepatotoxic Drugs" which may cause heightened response to anticoagulation drugs. All such considerations have been coded into the guideline. During guideline execution, the required data are retrieved from the EHR server through the "PCC-09 Care Management Data Query". In this case, the careProvisionCode in the subscription request is specified as PROBLIST for problem 


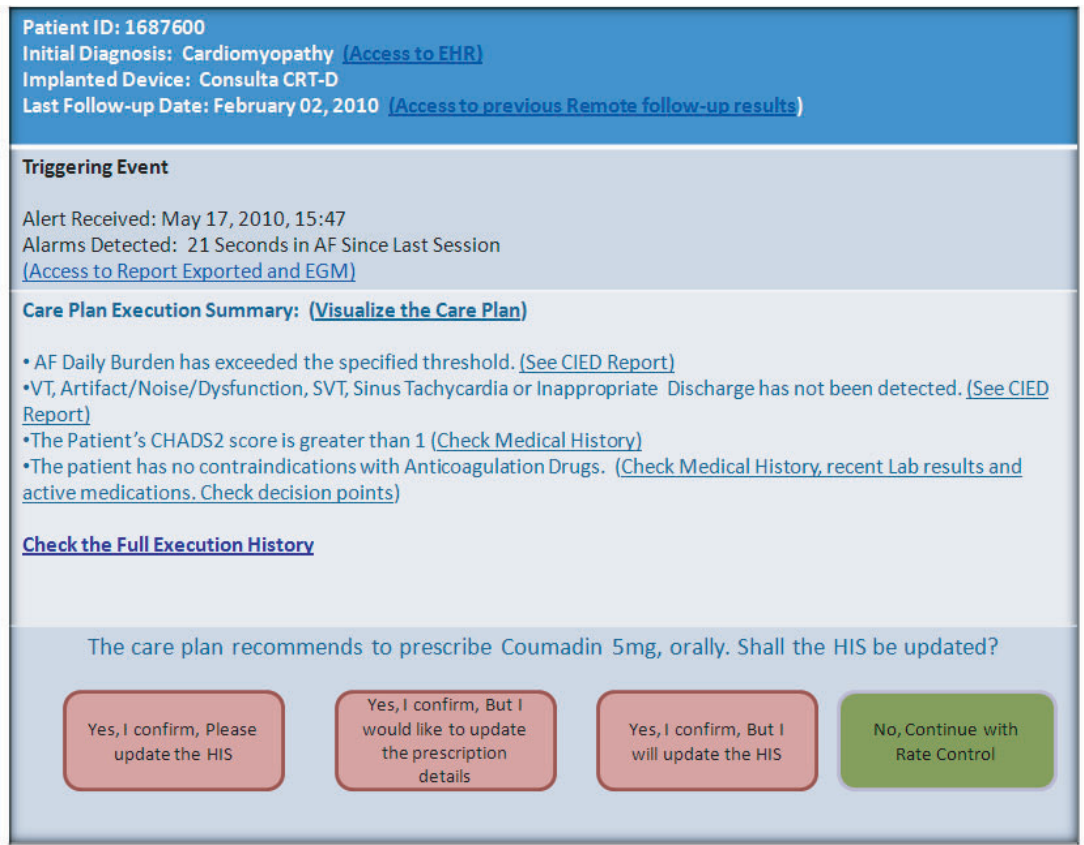

Figure 6. A sample recommendation window of care plan engine

concern list, as LABCAT for the lab results and as CURMEDLIST for the active medications. As a response, respective "PCC-10 V3 Care Management Update" messages are received and recorded in the care plan engine database. These values are checked while the care plan is executed to determine possible contraindications with anticoagulation drugs.

\subsection{Recommendations and Consultations with the Physician}

As mentioned in the previous section, the care plan engine checks the contraindications by accessing the EHR of the patient based on the guideline specified in the care plan, and if no contraindication is indicated, the care plan engine presents the "Anticoagulation Drug Prescription" recommendation (R14) to the physician through a graphical interface (as shown in Figure 6). This interface provides detailed information about the execution steps of the care plan: patient's initial diagnosis, information about the implanted device, and the link to the previous remote follow-up reports. The information about the triggering event for the care plan execution is then presented by allowing the physician to check the EGM and the reports generated by the CIEDs. Brief information about care plan execution steps is provided, and a link is given to a graphical monitoring tool which shows the care plan workflow and the results of each decision step such as the retrieved EHRs and the lab results. After the recommendation is presented to the physician, different options are provided to guide the care plan flow, 
such as updating the hospital information system for storing this prescription, or continuing with the rest of the care plan for evaluating the rate and rhythm control treatment options.

The care plan then continues with evaluating possible treatment options for "Heart Rate Problems", using data from patient's EHR. For example in the case of "AF NormaFrequent", checking the symptoms, the duration of the arrhythmia, the concomitant comorbidities and the medications of the patient, the care plan engine may recommend "to coordinate an urgent in person/remote or routine follow-up". Similarly in the case of "AF Tachycardia", checking the history of "heart failure" and the presence of "accessory pathway" from the EHR data extracted from the "Care Management Update" messages, the care plan engine may recommend alternative rate control drugs such as Digoxin, Amiodarone, Metoprolol, Propranolol, Diltiazem, Verapamil and Esmolol. Guidance on possible doses and major side effects can thus be presented to the physician in an informed manner. It should be noted that all the decisions recommended by the care plan engine need the physician's approval; the care plan engine can only assist the physician.

\section{DISCUSSION}

As presented in Section 1.1, a number of tele-monitoring systems provided by CIED manufacturers are available for remote monitoring of CIED Patients [11-19]. The iCARDEA interoperability architecture allows remote monitoring of CIED patients for early detection of possible medical problems and on top of the existing work, it enables seamless access to the EHRs of the patient so that individualized therapy recommendations can be proposed to the physicians.

The other systems enable transfer of CIED data periodically or upon an event to the data centers of the manufacturers for the physician's review of the events and CIED data analysis. However, decision making is only based on CIED data, and performed by the CIED manufacturer systems. After receiving the analysis of CIED data, when necessary (as explained in [18]), the physician manually accesses the EHRs of the patient and determines possible treatments and intervention options. The significance of the iCARDEA system presented in this paper is that it complements these procedures by providing a clinical-guideline-based decision making mechanism where not only CIED data but also EHRs of the patient are seamlessly accessed and analyzed to generate standardized guidance on the possible treatments and intervention options to assist the medical doctor in decision making.

Currently, the development of the iCARDEA system continues. After completing all the system components including the security and privacy measures, a clinical trial is planned. The aspects of evaluation and validation will include (a) the identification of possible medical risks which could not be detected but will be possible to detect through iCARDEA system; (b) time and pathway from patient's symptoms to clinical decision; (c) indicators on how remote iCARDEA monitoring can reduce risks for the patients (concerning the device and medical risks); (d) usability and acceptance of the iCARDEA system by patients and medical experts; (e) the contribution of the system to patients' well-being; (f) current barriers and limits for future developments; and (g) the 
adequacy of the iCARDEA security and privacy measures. Our future work also includes the development and automation of the flowcharts of two more care plans for the management of heart failure and ventricular tachycardia.

\section{CONCLUSION}

In this paper, we have presented the interoperability architecture of iCARDEA system enabling remote monitoring of CIED patients through computerized clinical guidelines via accessing the clinical details of the patient from EHR Systems and the CIED devices seamlessly. We have demonstrated that this interoperability architecture enables remote monitoring of AF patients through CIED devices by an example care plan execution scenario. Different from the existing remote monitoring systems in the literature, iCARDEA supports the physicians in the decision making process by executing the care plan, accessing the past medical history and current status of the patient when necessary through standard interfaces proposed by industry initiatives such as IHE IDCO and IHE $\mathrm{CM}$ profiles. Through iCARDEA, early protective interventions will be enabled upon the detection of asymptomatic clinical events such as AF through CIEDs. Automated care plan execution is also particularly useful for physicians who do not have the expertise of a cardiac electro-physiologist, but need to coordinate the remote follow-up of CIED Patients.

\section{ACKNOWLEDGEMENTS}

The research leading to these results has received funding from the European Community's Seventh Framework Programme (FP7/2007-2013) under grant agreement no. ICT-248240, iCARDEA Project.

\section{REFERENCES}

[1] HRS/EHRA Expert Consensus on the Monitoring of Cardiovascular Implantable Electronic Devices (CIED): Description of Techniques, Indications, Personnel, Frequency and Ethical Considerations

[2] Remote, Wireless, Ambulatory Monitoring of Implantable Pacemakers, Cardioverter Defibrillators, and Cardiac Resynchronization Therapy Systems, A. Lazarus, Pacing and Clinical Electrophysiol. 2007 Jan;30 Suppl 1, 2-12

[3] iCARDEA Project, An Intelligent Platform for Personalized Remote Monitoring of the Cardiac Patients with Electronic Implant Devices, http://www.icardea.eu/. Last accessed October 15, 2010.

[4] IHE Implantable Device - Cardiac - Observation Profile, http://www.ihe.net/Technical_Framework/ upload/IHE_PCD_TF_Supplement_IDCO_2009-08-10.pdf. Last accessed October 15, 2010.

[5] IHE Patient Care Coordination (PCC) Technical Framework Supplement, 2008-2009, Care Management (CM), Draft for Trial Implementation, August 22, 2008

[6] Institute of Medicine, Crossing the Quality Chasm: A New Health System for the 21st Century, National Academy Press, Washington DC, 2001

[7] Valentin Fuster FESCet al ACC/AHA/ESC 2006 guidelines for the management of patients with atrial fibrillation, in : Europace 2006

[8] Wilkoff, Bruce / Auricchio, Angelo / Brugada, Josep / Cowie, Martin / Ellenbogen, Kenneth / Gillis, Anne / Hayes, David / Howlett, Jonathan / Kautzner, Josef / Love, Charles / Morgan, John / Priori, Silvia / Reynolds, Dwight / Schoenfeld, Mark / Vardas, Panos: HRS/EHRA Expert Consensus on the Monitoring of Cardiovascular Implantable Electronic Devices (CIEDs): Description of Techniques, Indications, Personnel, Frequency and Ethical Considerations, in: Europace 2008 10(6):707-725; doi:10.1093/europace/eun122 
[9] Computer-based medical guidelines and protocols: A primer and current trends, eds A. Ten Teije, S. Miksch, P. Lucas, IOS Press, Amsterdam, 2008

[10] Boxwala AA, Peleg M, Tu S et al. GLIF3: a representation format for sharable computer-interpretable clinical practice guidelines. Journal of Biomed Inform., 2004, 37(3), 147-61.

[11] Sticherling C, Kühne M, Schaer B, Altmann D, Osswald S., Remote monitoring of cardiovascular implantable electronic devices: prerequisite or luxury?, Swiss Med Wkly. 2009, 139(41-42), 596-601.

[12] Egon Toft, Implantable electrocardiographic monitoring-clinical experiences, Journal of Electrocardiology, 2006, 39, 47-S49

[13] Scholten MF, Thornton S, Theuns DA, Res J, Jordaens LJ. Twiddler's syndrome detected by home monitoring device. PACE 2004, 27:1151.

[14] Nielsen JC, Kottkamp H, Zabel M, Aliot E, Kreutzer U, Bauer A et al. Automatic home monitoring of implantable cardioverter defibrillators. Europace 2008, 10, 729-35.

[15] Boriani G, Diemberger I, Martignani C, Biffi M, Valzania C, Bertini M, Domenichini G, Saporito D, Ziacchi M, Branzi A., Telecardiology and remote monitoring of implanted electrical devices: the potential for fresh clinical care perspectives., J Gen Intern Med. 2008 Jan;23 Suppl 1, 73-7

[16] Klersy C, De Silvestri A, Gabutti G, Regoli F, Auricchio A. A meta-analysis of remote monitoring of heart failure patients J Am Coll Cardiol. 2009, 54, 1683-1694

[17] Haran B. and David S., Remote monitoring and follow-up of pacemakers and implantable cardioverter defibrillators, Europace 2009, 11, 701-709

[18] Koos R, Sinha AM, Stellbrink C. Home monitoring in an ICD patient with incessant tachycardia. Z Kardiol.2005, 94:461.

[19] Ellery S, Pakrashi T, Paul V, Sack S. Predicting mortality and rehospitalization in heart failure patients with home monitoring: the home CARE pilot study. Clinical Research in Cardiology,2006, Volume 95, Supplement 3, 29-35

[20] ESC guidelines for the diagnosis and treatment of acute and chronic heart failure 2008: the Task Force for the diagnosis and treatment of acute and chronic heart failure 2008 of the European Society of Cardiology

[21] HL7 Clinical Document Architecture (CDA), http://hl7.org/library/Committees/-structure/ CDA.ReleaseTwo.CommitteeBallot03.Aug.2004.zip. Last accessed October 15, 2010.

[22] IHE Care Coordination Framework, Content Modules, http://wiki.ihe.net/index.php?title= 1.3.6.1.4.1.19376.1.5.3.1.1\#Medical_Documents_Specification_1.3.6.1.4.1.19376.1.5.3.1.1.1. Last accessed October 15, 2010.

[23] IHE Cross Enterprise Document Sharing (XDS) Profile, http://www.ihe.net/-Technical_Framework/ index.cfm\#IT. Last accessed October 15, 2010.

[24] Douglas P Zipes et al. ACC/AHA/ESC guidelines for the management of patients with ventricular arrhythmia and the prevention of sudden cardiac death, in: Europace 2008

[25] "Risk of Stroke with AF". VA Palo Alto Medical Center and at Stanford University: the Sports Medicine Program and the Cardiomyopathy Clinic. http://www.cardiology.org/tools/risk_of_ stroke_AF.html. Last accessed December 07, 2010.

[26] IHE History of Past Illness Section, http://wiki.ihe.net/index.php?title-=1.3.6.1.4.1.19376.1.5.3.1.3.8. Last accessed October 15, 2010.

[27] IHE Problem Concern Entity Template, http://wiki.ihe.net/index.php?title-=1.3.6.1.4.1.19376.1.5.3.1.4.5.2. Last accessed October 15, 2010. 


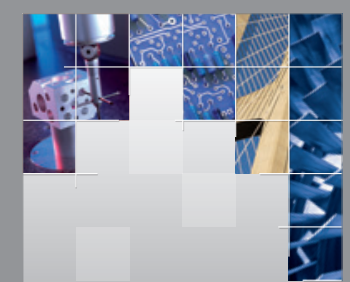

\section{Enfincering}
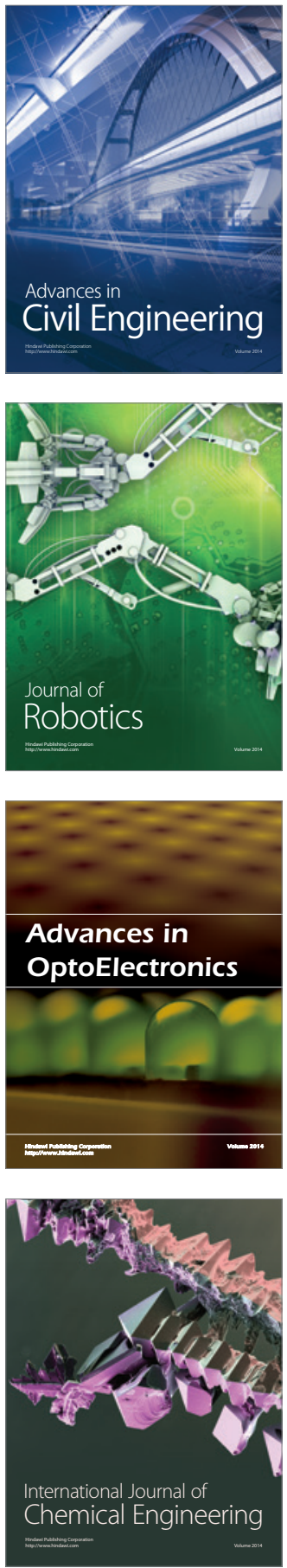

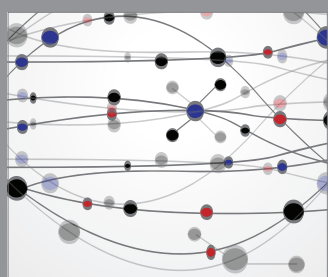

The Scientific World Journal

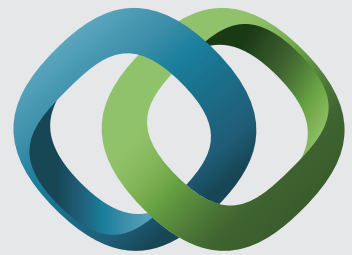

\section{Hindawi}

Submit your manuscripts at

http://www.hindawi.com
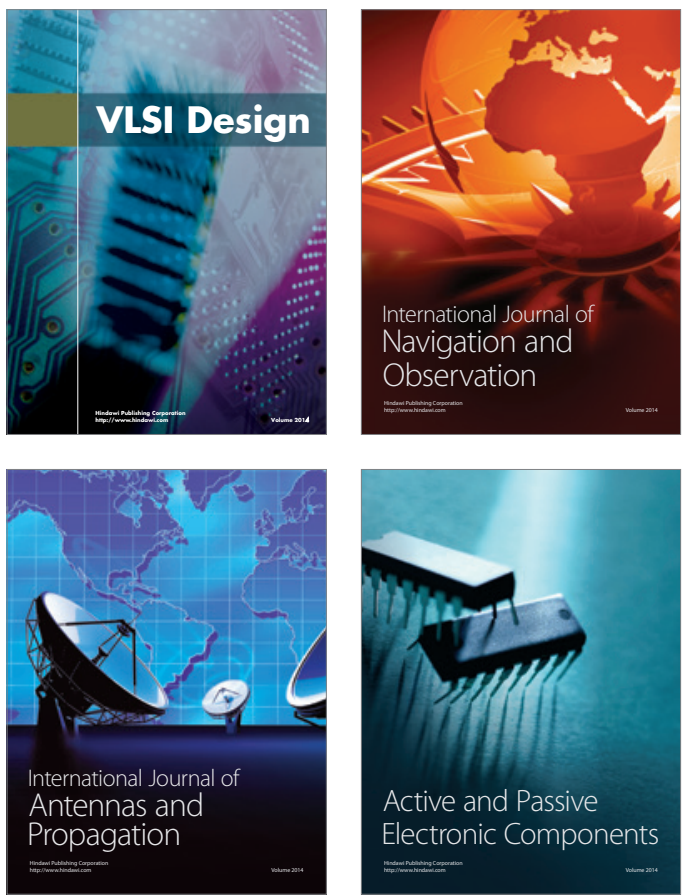
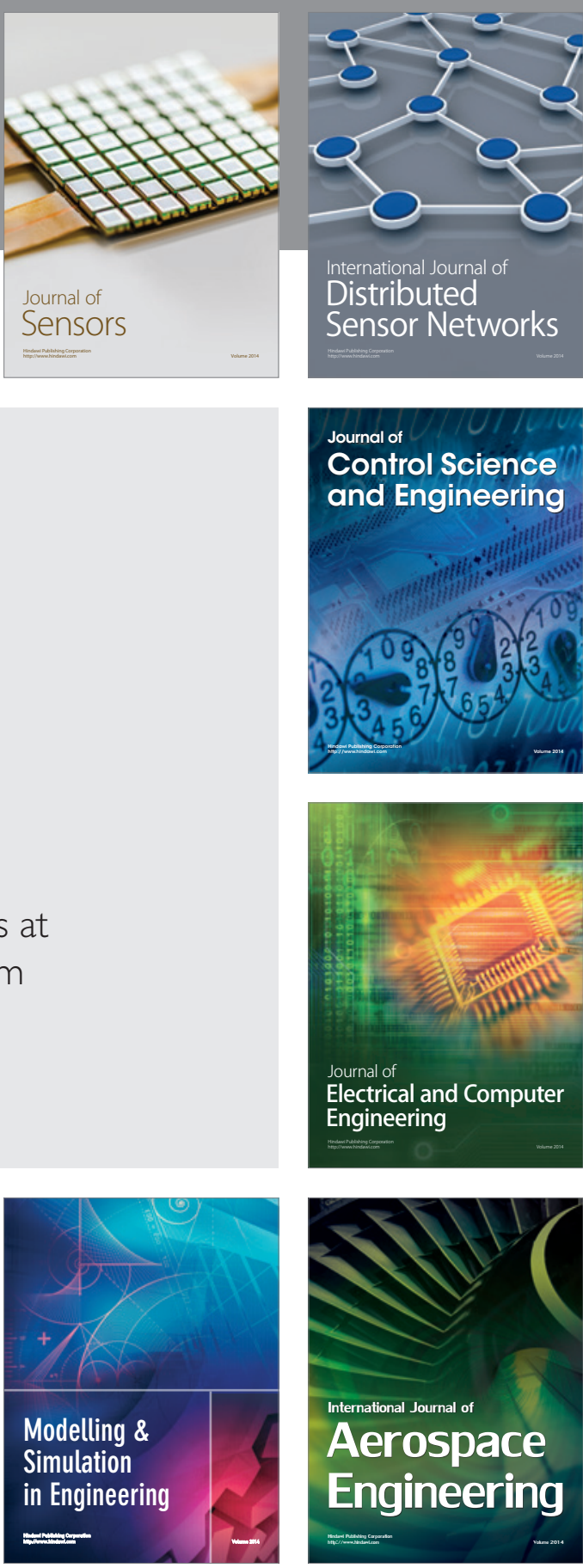

International Journal of

Distributed

Sensor Networks

Journal of

Control Science

and Engineering
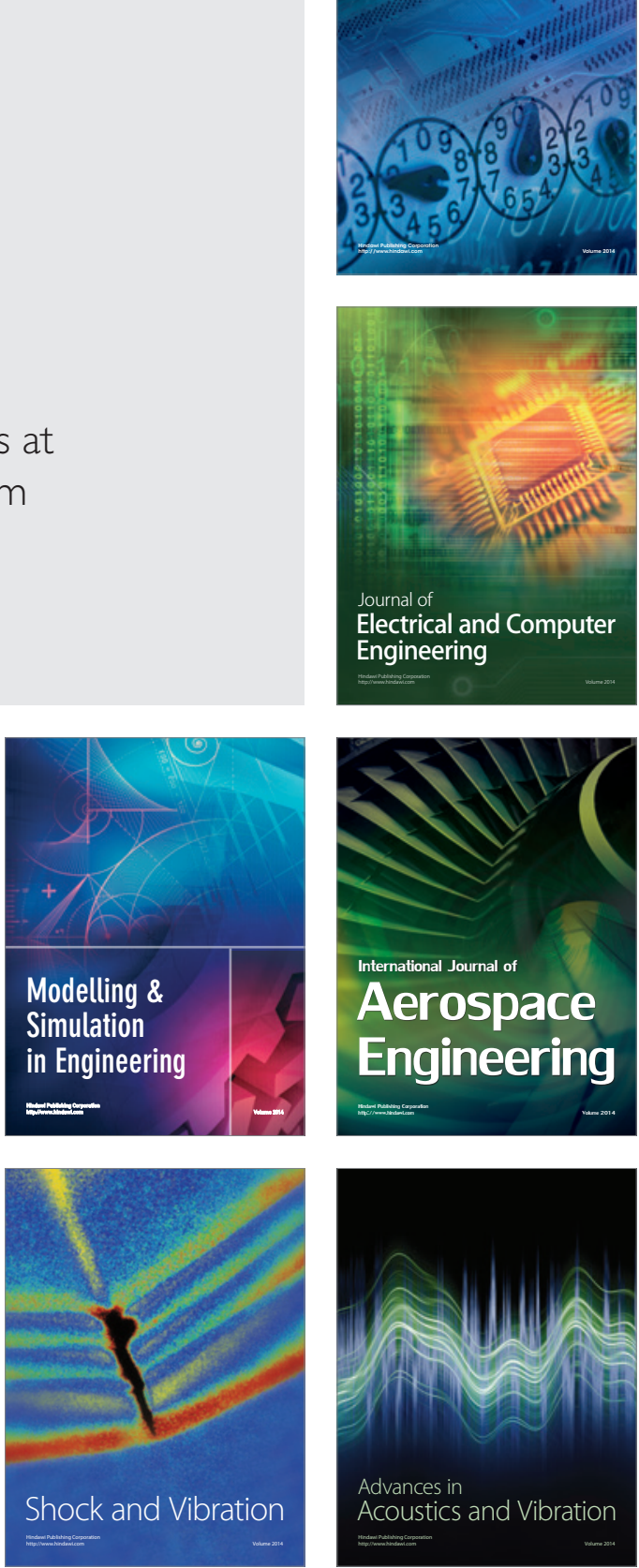